\title{
BACK TO THE BASICS- UTILITY OF THE DISC DAMAGE LIKELIHOOD SCALE FOR OPTIC DISC EVALUATION IN GLAUCOMA
}

\author{
Valerie Menezes ${ }^{1}$, Ugam P. S. Usgaonkar ${ }^{2}$
}

${ }^{1}$ Senior Resident, Department of Ophthalmology, Goa Medical College, Bambolim, Tiswadi, Goa.

2 Professor and HOD, Department of Ophthalmology, Goa Medical College, Bambolim, Tiswadi, Goa.

\begin{abstract}
\section{BACKGROUND}

Glaucoma is a progressive disease and requires serial evaluation of the optic disc to monitor progression and optimize therapy. The Disc Damage Likelihood Scale (DDLS) a new system for assessing disc damage in glaucoma, is a simple but versatile tool that needs only clinical skills and minimal instrumentation. The DDLS could be especially useful in those parts of India where sophisticated technological equipment like Heidelberg Retinal Tomography and Optical Coherence Tomography are not available.

The aim of this study was to estimate the reliability of the DDLS and to compare the sensitivity and specificity of the DDLS, Vertical Cup to Disc Ratio (VCDR), Mean Deviation (MD) of the Visual Field (VF) and the Hodapp-Parish-Anderson (HPA) System in distinguishing between normal and glaucomatous eyes.
\end{abstract}

\section{MATERIALS AND METHODS}

In this cross-sectional study, patients of Primary Open Angle Glaucoma were enrolled over 2 years. The study group had 110 eyes of 60 patients and control group had 40 eyes of 20 normal subjects. 10 eyes were excluded due to advanced glaucoma. Disc Damage was assessed by the VCDR and DDLS using slit lamp biomicroscopy with 90D lens and fundus photographs. VF were graded using the HPA system. Pearsons one-tailed correlation test was used to find the correlation between DDLS, MD, Pattern Standard Deviation (PSD) and HPA system. Receiver Operating Characteristic curves were drawn.

\section{RESULTS}

Mean patient age: 55 years. MD of VF -7.83. DDLS had highest Area under curve (AUC) of 0.925 followed by VCDR (AUC=0.886), HPA $(A U C=0.850)$ and MD $(A U C=0.834)$ in the separation of glaucoma patients from normal. DDLS was strongly co-related with MD ( $r=-0.768, p<0.0001)$, PSD ( $r=0.706, p<0.0001)$ and HPA $(r=0.699, p<0.0001)$. The co-relation between the VCDR and the HPA system was lower $(\mathrm{r}=0.620, \mathrm{p}<0.0001)$.

\section{CONCLUSION}

DDLS is more sensitive than conventional Cup to Disc Ratio for disc evaluation in patients of POAG and co-relates accurately with visual field loss and hence may be especially valuable in situations where more sophisticated machines are unavailable.

\section{KEYWORDS}

Disc Damage Likelihood Scale, Glaucoma.

HOW TO CITE THIS ARTICLE: Menezes V, Usgaonkar UPS. Back to the basics- utility of the disc damage likelihood scale for optic disc evaluation in glaucoma. J. Evolution Med. Dent. Sci. 2017;6(8):653-658, DOI: 10.14260/Jemds/2017/140

\section{BACKGROUND}

Glaucoma is the second leading cause of blindness worldwide. ${ }^{1}$ Primary Open Angle Glaucoma (POAG) can be considered a chronic, progressive anterior optic neuropathy that is accompanied by a characteristic cupping and atrophy of the optic disc, visual field loss, open angles and no obvious ocular or systemic conditions.

Since POAG is very slowly progressive, it is usually asymptomatic until late in its course and affected individuals can develop severe damage before they seek professional help. ${ }^{2}$

Financial or Other, Competing Interest: None.

Submission 21-12-2016, Peer Review 14-01-2017,

Acceptance 20-01-2017, Published 25-01-2017.

Corresponding Author:

Dr. Valerie Menezes,

Siddarth Apartments,

\#2/S-2, Tonca, P. O.,

Caranzalem-403002.

E-mail: vlm1142@gmail.com

DOI: $10.14260 /$ jemds $/ 2017 / 140$
As the visual impairment caused by glaucoma is irreversible, early detection is essential.

Generally, changes in Optic Nerve Head (ONH) morphology, precede the progress of visual field defects. Morphological changes in the $\mathrm{ONH}$ are therefore considered important early biomarkers of Glaucomatous Optic Neuropathy and its progression. ${ }^{3}$ While perimetry may be easy to use, it may overlook early warning signs of glaucoma.

A thorough meticulous clinical examination of the optic disc remains the cornerstone of glaucoma diagnosis.

Currently disc evaluation is often inadequate because of reliance on cup to disc ratios and on the results of imaging technology. 4

The most commonly used quantitative classification of the optic nerve is the cup to disc ratio which describes the disc using the cup diameter as a percentage of the overall disc diameter. The fact that the system does not account for disc size and that focal neuroretinal thinning is not adequately highlighted, limits its diagnostic accuracy. ${ }^{4}$

With the advancement in technology, several sophisticated and expensive diagnostic machines like confocal laser scanning ophthalmoscopy (Heidelberg retinal tomograph -HRT), scanning laser polarimetry and Optical 
Coherence Tomography (OCT) have been introduced to detect glaucomatous nerve damage. Information from imaging devices should be considered complementary and not a substitute to clinical examination. 5 Moreover in developing countries like India, these machines and often unavailable or inaccessible for majority of the population. Hence a thorough clinical examination of the optic nerve head remains invaluable.

In this study, we evaluated the reliability of a new scale, the Disc Damage Likelihood Scale (DDLS) by co-relating its stages with visual field changes in cases of Primary Open Angle Glaucoma. The disc damage likelihood scale (DDLS) devised by Spaeth et al, as a new method for estimating the amount of disc damage in patients with glaucoma, is based on the appearance of the neuroretinal rim corrected for disc diameter. 6

The study compares the utility of the DDLS with conventional Cup to Disc Ratio for disc assessment in patients of POAG.

\section{The Objectives of the Study were}

1. To estimate the Reliability of the Disc Damage Likelihood Scale (DDLS) by correlating its stages with Visual Field changes in cases of Primary Open Angle Glaucoma.

2. To compare the sensitivity and specificity of the DDLS, Vertical Cup to Disc Ratio (VCDR), Mean Deviation (MD) of the Visual Field (VF) and the Hodapp-Parish-Anderson Scoring System in distinguishing between normal and glaucomatous eyes.

\section{MATERIALS AND METHODS}

This cross-sectional study was undertaken with the approval by the Institutional Legal Ethics Committee.

In the study, patients above the age of 40 years, already diagnosed to have POAG and on treatment for a considerable period of time as well as newly diagnosed patients of POAG at a tertiary care centre were enrolled from October 2011 to September 2013.

In all, 150 eyes of 80 patients were enrolled. 110 eyes of 60 patients of POAG were included in the study group and 40 eyes of 20 normal subjects were included as controls. 10 eyes were excluded on account of advanced glaucoma as VF assessment was not possible.

All patients were subjected to detailed history and thorough ophthalmological examination including:

- Anterior Segment Examination.

- Visual Acuity Assessment and Refraction-Best corrected Visual Acuity (BCVA) was applied to each patient according to refraction.

- IOP measurement using Goldmann applanation tonometry.

- Gonioscopy using Goldmann 1- mirror lens.

- Dilated Fundus Examination- by Slit Lamp Examination using Volk 90D Lens. Optic Disc Photography using Canon CF-1 Digital Fundus Camera was done.

- VF Assessment using Standard Automated PerimetrySwedish Interactive Threshold Algorithm (SITA) 30-2 program on Humphrey Field Analyser.

Exclusion Criteria were: Advanced Glaucoma in which VF assessment was not possible; Secondary, Angle Closure and Congenital Glaucoma; Refractive Error of more than 5
Dioptres from emmetropia or $>2.5$ Dioptres of Astigmatism; History of intraocular trauma or neurological diseases; Patients of POAG with unreliable VF (Fixation Losses $>20 \%$ or False Positive $>15 \%$ or False Negatives $>15 \%$ ) were excluded.

Normal Eyes were defined as those with no family history of glaucoma in a first degree relative, open angles on gonioscopy, having a BCVA of $6 / 12$ or better with refractive error between +3.00D and -5.00 Dioptres, Normal appearing Optic Nerve Head and Normal Visual Fields.

Glaucomatous eyes had open angles on gonioscopy, evidence of glaucomatous optic neuropathy and abnormal VF tests. Glaucomatous optic neuropathy was defined as difference in vertical cupping more than 0.2 between eyes, rim thinning, notching or RNFL defects.

An abnormal VF test was defined as an abnormal Glaucoma Hemifield test (outside normal limits) and at least 3 non-edge points in the same hemi field that exceeded a probability value of $0.5 \%$ in the pattern deviation plot. In addition, there were no other signs of optic nerve diseases or retinal diseases which could lead to the disc or field findings.

\section{Optic Disc Evaluation}

The VCDR was measured by comparing the diameter of the cup with that of the disc in the vertical axis using a Volk $90 \mathrm{D}$ lens at the slit lamp. An Optic Disc Photograph was taken and an Automated Measurement of VCDR was made. For each disc, the estimate of VCDR was made based on the average of the above two measurements.

Presence of notching, disc haemorrhages, $\beta$ zone parapapillary atrophy and retinal nerve fibre layer defects were noted.

\section{For the DDLS Staging}

1. Vertical Disc Diameter was measured using Volk 90D Lens with the length of the slit beam adjusted to match the Vertical Disc Size. The Vertical Disc Size was then read from the scale of the Slit Lamp and multiplied by a correction factor of 1.33 .

2. Rim to Disc Ratio was calculated by comparing the narrowest radial width of the Neuroretinal Rim with that of the Disc Diameter in the same axis. When there was no rim remaining, the Rim to Disc Ratio was taken as 0 and the circumferential extent of Rim Absence measured in degrees.

The Automated Measurement of Rim to Disc Ratio on the optic disc photograph was noted.

DDLS Stage was assigned to each Disc based on the average of the above two measurements using the DDLS Normogram. ${ }^{7}$

\section{Visual Field Analysis}

It was done using SITA 30-2 Program on Humphrey's Visual Field Analyser with a Goldmann Size III $\left(0.43^{\circ}\right)$ stimulus on a 31.5 Apostil background.

VF were analysed and graded using the Hodapp, Parish and Anderson's Classification. ${ }^{8}$

\section{Statistical Analysis}

Pearson one- tailed correlation test was used to explore the correlation between the DDLS Stage, VCDR and the Visual 
Field Indices i.e. Mean Deviation (MD) and Pattern Standard Deviation (PSD).

Scatter plots were obtained for the relation of DDLS with the field MD and PSD.

The Receiver Operating Characteristic (ROC) curves were drawn and the Area under the Curve (AUC) was used as a statistic in non-parametric analysis to estimate the value of each method in identifying glaucomatous eyes. All tests were two tailed and a 5\% significance level was maintained. The procedure of analysis program SPSS was used.

\section{RESULTS}

The study group consisted of people with an average age of 55 years. There were 43 males and 37 females included in the study.

\section{Visual Field}

Mean Deviation: -7.83(SD: 7.44).

Range: -30 to 2.00 .

\section{Optic Nerve Parameters}

Mean VCDR: 0.57(SD -.0.15).

Clinical Examination using DDLS had the highest Area under Curve (AUC) of 0.925 , predictive value of variables in the separation of glaucoma patients from normal.

This was followed by VCDR, HPA scoring and MD on VF examination (Table/Figure 1 ).

ROC curves were drawn (Table/Figure 2).

Scatter plots were obtained for the relation of DDLS with field MD and PSD (Table / Figure 3a and 3b)

Histogram showing the distribution of the DDLS stages and their corresponding Hodapp-Parish-Anderson (HPA) visual field stages (Table /Figure 4)

Table/Figure 5 shows one of the fundus photographs of the optic disc with the DDLS Stage, taken during the study and corresponding Visual Fields.

\begin{tabular}{|c|c|c|c|c|c|}
\hline \multirow{2}{*}{$\begin{array}{c}\text { Test } \\
\text { Result } \\
\text { Variables }\end{array}$} & \multirow[t]{2}{*}{ Area } & \multirow{2}{*}{$\begin{array}{c}\text { Std. } \\
\text { Error* }\end{array}$} & \multirow{2}{*}{$\begin{array}{c}\text { Asymptotic } \\
\text { Sig.+ }\end{array}$} & \multicolumn{2}{|c|}{$\begin{array}{l}\text { Asymptotic } 95 \% \\
\text { Confidence } \\
\text { Interval }\end{array}$} \\
\hline & & & & $\begin{array}{l}\text { Lower } \\
\text { Bound }\end{array}$ & $\begin{array}{l}\text { Upper } \\
\text { Bound }\end{array}$ \\
\hline VCDR & .886 & .026 & .000 & .834 & .938 \\
\hline DDLS & .925 & .020 & .000 & .886 & .965 \\
\hline HPA & .850 & .030 & .000 & .791 & .909 \\
\hline MD1 & .832 & .032 & .000 & .769 & .895 \\
\hline \multicolumn{6}{|c|}{$\begin{array}{c}\text { Table/Figure 1. Area under Curve for Receiver Operator } \\
\text { Characteristics for VCDR (Vertical cup to Disc Ratio), } \\
\text { DDLS (Disc Damage Likelihood Scale), HPA } \\
\text { (Hodapp-Parish-Anderson) score and MD1 } \\
\text { (Mean Deviation) of the Visual Field }\end{array}$} \\
\hline
\end{tabular}

The test result variable(s): VCDR, DDLS, HPA, MD1 has at least one tie between positive actual state group and the negative actual state group.

*Under the nonparametric assumption.

+Null Hypothesis: True Area= 0.5 .

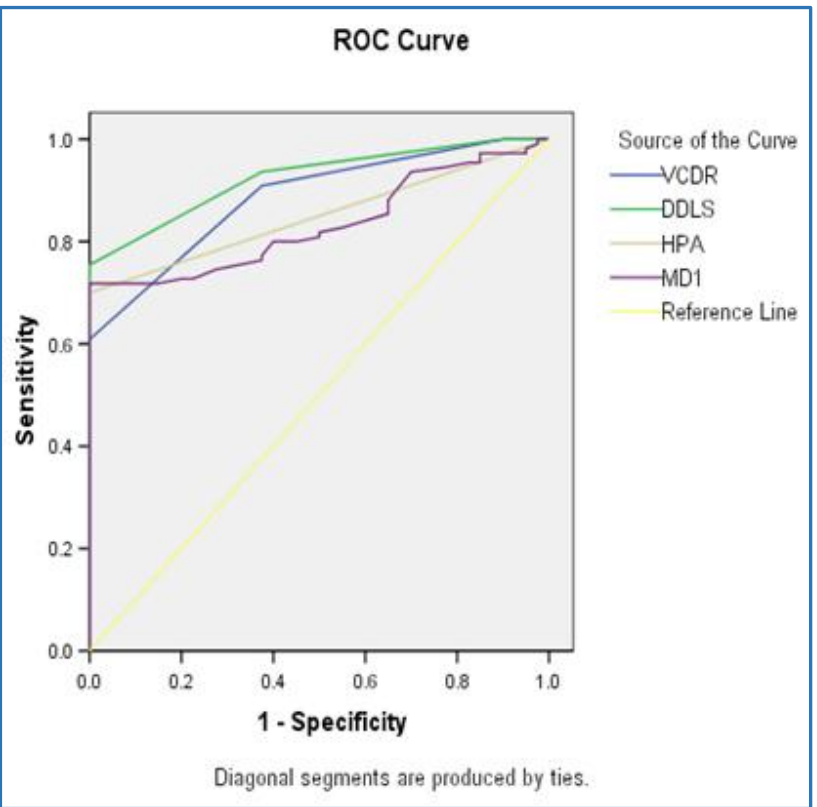

Table/Figure 2. Showing Receiver operating characteristic curves for Disc Damage Likelihood Score (green line), Vertical Cup to Disc Ratio (blue line), Hodapp-Parish Anderson scoring (brown line) and Mean Deviation (purple line) for glaucoma patients versus normal subjects

DDLS: Disc Damage Likelihood Scale.

VCDR: Vertical Cup to Disc Ratio.

HPA Score: Hodapp-Parish-Anderson Score.

MD1: Mean Deviation.

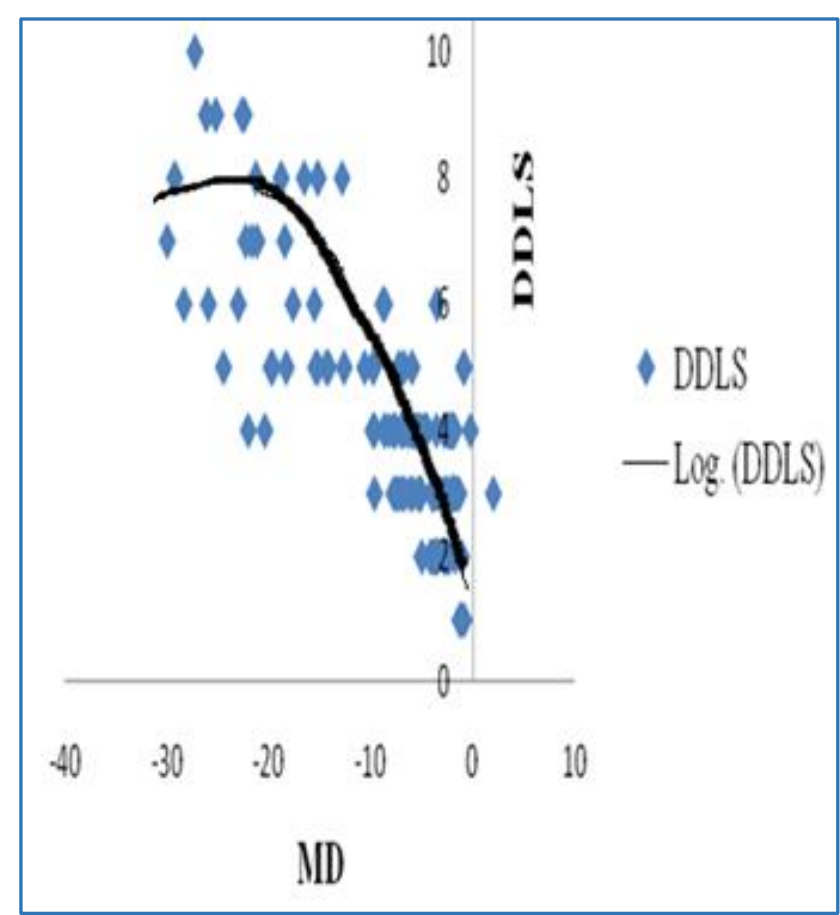

Table/Figure 3a. Scatter plot of relation between MD of the Visual Field and DDLS

Linear regression showed a statistically significant correlation, with a correlation coefficient. $\mathrm{r},=-0.768, \mathrm{p}<0.0001$. 


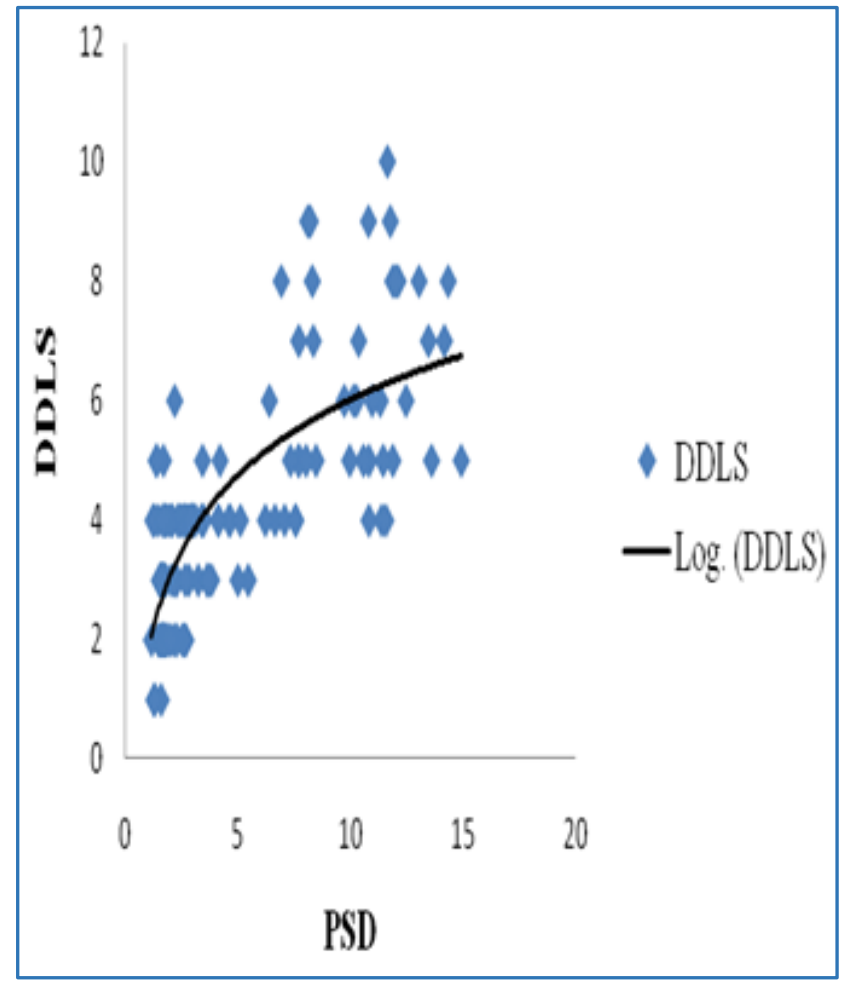

Table/Figure 3b. Scatter plot of relation between PSD of the visual field and DDLS

Linear Regression Analysis showed a statistically significant correlation for DDLS with a correlation coefficient $\mathrm{r}=0.706, \mathrm{p}<0.0001$.

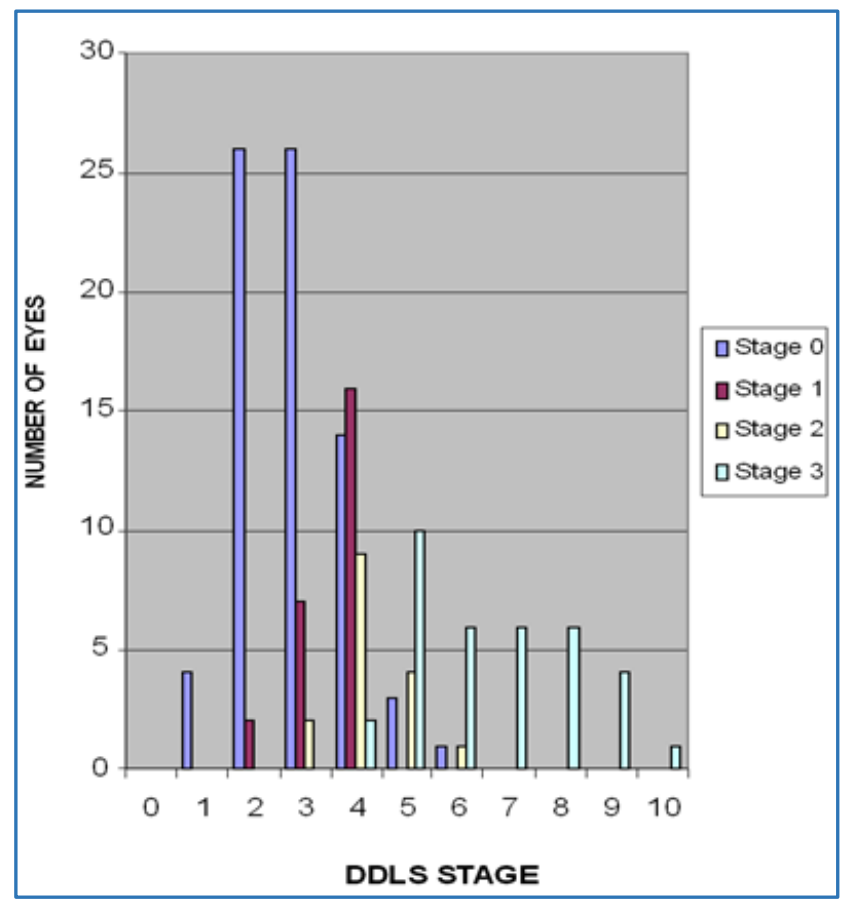

Table/Figure 4. Histogram showing the distribution of the DDLS Stages and their corresponding HodappParish-Anderson (HPA) visual field stages. The Vertical Axis shows the number of eyes

From the above graph, it was seen that optic discs staged as DDLS 0-3 were not associated with any visual field defects or with Stage 1 (early) defects. Discs staged as DDLS 4-6 were usually associated with Stage 1, Stage 2 (Moderate) or Stage 3 defects. Discs graded as DDLS 7-10 were exclusively associated with Stage 3 (Severe defects).

This graph emphasizes that there is a good co-relation between the DDLS Staging system and the HPA system.
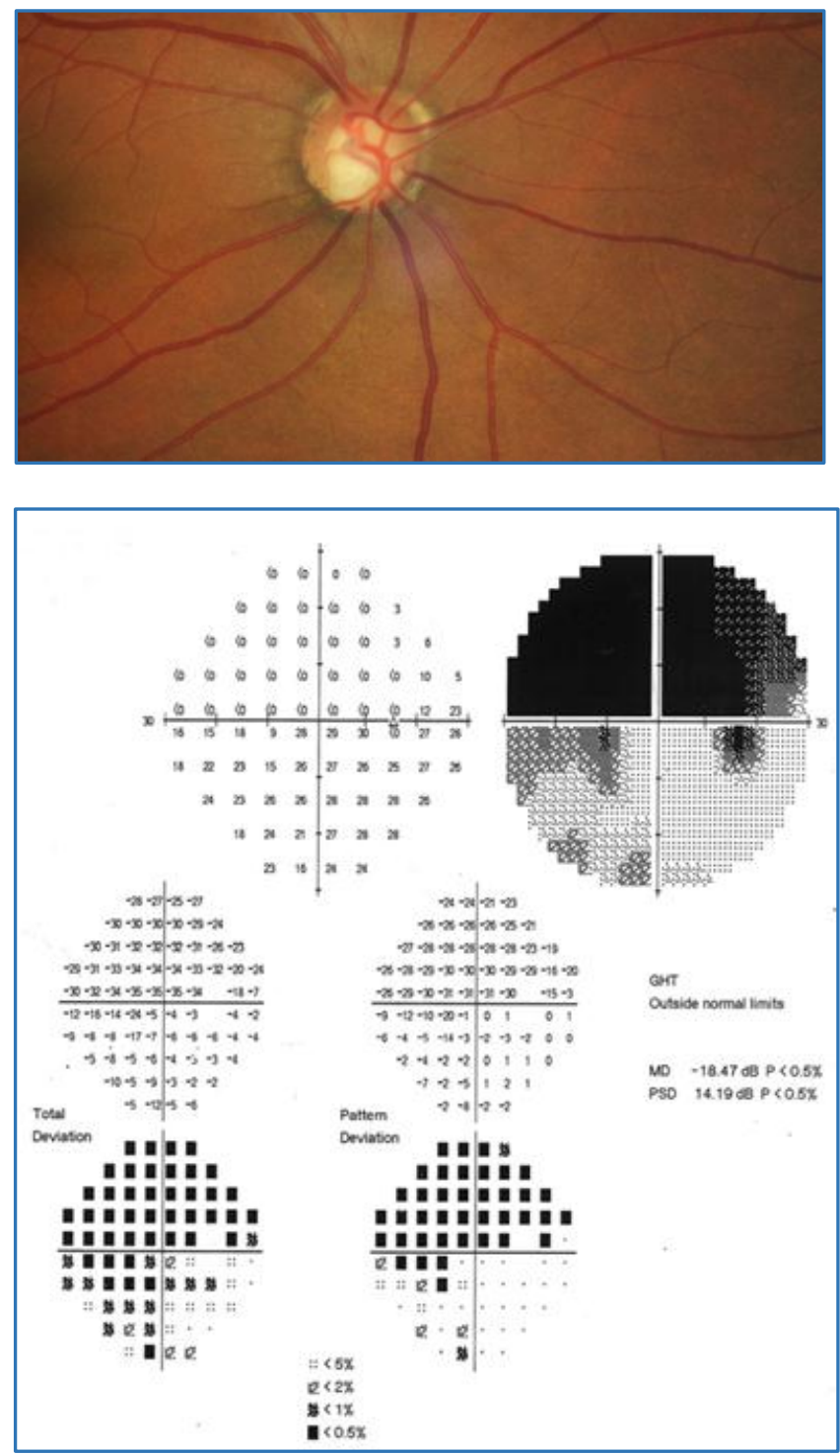

Table/Figure 5. Right eye of a patient showing severe glaucomatous visual field loss (Below) and corresponding fundus photograph showing a VCDR of 0.8 with inferior notching and DDLS Stage 7 in a medium sized disc

\section{DISCUSSION}

The results in this study show that DDLS is a very good method to distinguish between glaucomatous and normal eyes.

Clinical examination using the DDLS had the highest Area under Curve $(A U C=0.925)$ predictive value of variables in the separation of glaucoma patients from normal patients. This was followed by the Vertical Cup to Disc Ratio (AUC=0.886), Visual Field HPA Score (AUC $=0.850$ ) and the Visual Field Mean Deviation (AUC=0.834) (Table /Fig. 2).

Among the four parameters evaluated in this study (i.e. DDLS, VCDR, HPA score and MD of the VF), the DDLS was found to be the best method to distinguish glaucoma patients from normal.

There was a strong inverse correlation between the DDLS and MD (Linear regression showed a statistically significant 
correlation, with a correlation coefficient $r$, $=-0.768$, $\mathrm{p}<0.0001$ ) and a strong positive correlation between the DDLS stage and PSD (Linear Regression Analysis showed a statistically significant correlation with a correlation coefficient $r=0.706, p<0.0001$ ).

(Table/Figure $3 \mathrm{a}$ and $3 \mathrm{~b}$ ).

The co-relation coefficient between the Vertical C/D Ratio and the HPA scoring was lower, $(r=0.620, p<0.0001)$ than that between the DDLS and the HPA system ( $r=0.699$, $\mathrm{p}<0.0001$ ) thereby showing that DDLS co-relates more accurately with VF loss than the VCDR.

The Cup to Disc (C/D) Ratio was devised by Armaly ${ }^{9}$ for estimating disc damage. The VCDR is a better measure of deviation from normal than the horizontal ratio because the early neuroretinal rim loss occurs preferentially at the upper and lower poles of the disc. 10

The C/D ratio has several shortcomings. It only indirectly examines the neuroretinal rim, by measuring the width of the cup rather than the surrounding neuroretinal rim tissue that determines its border. Since the VCDR is calculated as the ratio of the cup to the disc in the vertical axis, focal thinning in an oblique axis can be overlooked. The C/D ratio does not take into consideration the optic disc size. Hence larger discs which are likely to have a larger C/D ratio (But may have normal neuroretinal rims) are more likely to be classified as glaucomatous, while small discs with small C/D ratio are more likely to be classified as normal whether they have actual glaucoma or not. ${ }^{11}$ The Cup to Disc Ratio has only limited value in the identification of glaucoma, in part because of the wide variation in the size of the disc and consequently of the cup in the general population. ${ }^{12}$

The fact, then, is that a relationship between C/D ratio and glaucoma combined with the ease of estimating the C/D ratio, resulted in the $\mathrm{C} / \mathrm{D}$ ratio becoming the standard way to describe the optic disc. ${ }^{13}$ The reproducibility of this system is only moderate. 6

Neuroretinal rim area is the disc parameter that is most highly correlated with the visual field. ${ }^{14}$ It is shown to be superior to the $\mathrm{C} / \mathrm{D}$ ratio method in its correlation with visual function and its ability to differentiate among normal eyes, eyes with suspected glaucoma and eyes with early glaucoma.14,15 The prediction of field loss in a glaucomatous eye is based on identifying sites from which neuroretinal rim tissue has been lost. This is seen as thinning, absence or pallor of the neuroretinal rim tissue. The Disc Damage Likelihood Scale incorporates this concept into its classification scheme using the radial width of the neuroretinal rim tissue measured at its thinnest point, the unit of measurement being the rim/disc ratio. ${ }^{14}$

The DDLS initially had seven stages 6,14 which was later expanded to have ten stages. ${ }^{7}$

The two principal advantages of DDLS are that firstly Disc size is taken into consideration-Classification of discs as small, medium or large allows adjustment of the neuroretinal rim thickness and reduces the misclassification based on disc size. Also by calculation of the rim to disc ratio, the DDLS focuses attention on how much neuroretinal rim tissue is present. ${ }^{11}$

DDLS has some limitations. It is possible that a patient with a static DDLS grade may have advancing damage-for example, if focal notching of the disc was followed by generalized atrophy. ${ }^{11}$ However, detailed drawing of the optic disc at every visit and longitudinal follow-up with other modalities help to overcome this limitation ${ }^{16}$ not all discs can be graded with the DDLS system. Many myopic and anomalous disks cannot be reliably fit into the DDLS system. For most optic nerve heads however, a DDLS grade can be accurately estimated within plus/minus one stage ${ }^{7}$ DDLS does not offer a system to document progression in more than one region or a new region of the optic disc until it is more severely involved than the originally documented area $^{11}$

A study by Danesh-Meyer et al ${ }^{11}$ to evaluate the relative diagnostic strength of C/D ratio, clinical DDLS, and Heidelberg retina tomograph (HRT-II) in patients with glaucoma, glaucoma suspects, and normal controls concluded, that the DDLS appears to be superior to $\mathrm{C} / \mathrm{D}$ ratio as a clinical approach to optic disc evaluation and that it is as sensitive and specific as laser imaging by HRT-II, at lower cost.

Several other studies ${ }^{17,18}$ have also shown that clinical evaluation of the Optic Nerve Head using the DDLS provides information that correlates well with $\mathrm{VF}$ and with data obtained from HRT.

Studies ${ }^{16,19,20}$ have shown that the DDLS has a close corelation with the VF and OCT parameters for glaucoma diagnosis.

A study by Greaney et al ${ }^{21}$ showed that imaging devices are no better than qualitative assessment of the optic disc stereo photographs by experienced observers at distinguishing normal eyes from those with early to moderate glaucoma.

Since the DDLS divides glaucomatous progression into 10 stages, it is better able to monitor disease progression than the VCDR. DDLS scores of 1-3 are rarely associated with glaucomatous visual field loss. Visual field loss usually will not occur before Stage 5 and one may choose to defer treatment and observe such patients closely. Unless glaucomatous progression has stabilized, a DDLS score of 6 through 10 strongly supports aggressive treatment. ${ }^{22}$

Our study shows that a thorough clinical examination of the disc using DDLS, along with fundus photographs is a sensitive and valuable tool for disc evaluation in POAG especially where OCT and HRT are unavailable.

\section{CONCLUSION}

As glaucomatous disc changes, may precede visual field loss by several years, early detection of optic nerve changes by careful examination of the optic disc, is critically important in the early diagnosis and management of Primary Open Angle Glaucoma.

The DDLS was more strongly co-related with the HPA scale than the VCDR.

The DDLS staging system is superior to the Cup to Disc Ratio for evaluating optic disc changes in Primary Open Angle Glaucoma. This clinical approach would be especially valuable in those circumstances where HRT and OCT are unavailable.

\section{REFERENCES}

[1] Kingman S. Glaucoma is second leading cause of blindness globally. Bulletin of the World Health Organization 2004;82(11):887-8. 
[2] Stamper RL, Lieberman MF, Drake MV. BeckerShaffer's diagnosis and therapy of the glaucomas. $8^{\text {th }}$ edn. Elsevier Health Sciences 2009: pgs. 592.

[3] Yokoyama Y, Tanito M, Nitta K, et al. Stereoscopic analysis of optic nerve head parameters in primary open angle glaucoma: the glaucoma stereo analysis study. PloS One 2014;9(6):e99138.

[4] Zangalli C, Gupta SR, Spaeth GL. The disc as the basis of treatment for glaucoma. Saudi Journal of Ophthalmology 2011;25(4):381-7.

[5] Greenfield DS, Weinreb RN. Role of optic nerve imaging in glaucoma clinical practice and clinical trials. American Journal of Ophthalmology 2008;145(4):598-603.

[6] Spaeth GL, Henderer J, Liu C, et al. The disc damage likelihood scale: reproducibility of a new method of estimating the amount of optic nerve damage caused by glaucoma. Transactions of the American Ophthalmological Society 2002;100:181-6.

[7] Spaeth GL, Paulus A. The colored glaucoma graph and its use in caring for patients with glaucoma: a new system of management presented in three parts. Journal of Current Glaucoma Practice 2010;4(2):8390.

[8] Brusini P, Johnson CA. Staging functional damage in glaucoma: review of different classification methods. Survey of Ophthalmology 2007;52(2):156-79.

[9] Armaly MF. Optic cup in normal and glaucomatous eyes. Investigative Ophthalmology \& Visual Science 1970;9(6):425-9.

[10] Bhartiya S, Gadia R, Sethi HS, et al. Clinical evaluation of optic nerve head in glaucoma. Journal of Current Glaucoma Practice 2010;4(3):115-32.

[11] Danesh-Meyer HV, Gaskin BJ, Jayusundera T, et al. Comparison of disc damage likelihood scale, cup to disc ratio, and heidelberg retina tomograph in the diagnosis of glaucoma. British Journal of Ophthalmology 2006;90(4):437-41.

[12] Reis AS, Toren A, Nicolela MT. Clinical optic disc evaluation in glaucoma. European Ophthalmic Review 2012;6(2):92-7.
[13] Wei H, Zangalli C, Spaeth GL. Valid estimation of the appearance of the optic nerve: the case against using cup/disk ratios. Journal of Current Glaucoma Practice 2010;4(3):133-6.

[14] Bayer A, Harasymoycz P, Henderer JD, et al. Validity of a new disk grading scale for estimating glaucomatous damage: correlation with visual field damage. Am J of Ophthalmol 2002;133(6):758-63.

[15] Hitchings RA, Brown DB, Anderton SA. Glaucoma screening by means of an optic disc grid. Br J of Ophthalmol 1983;67(6):352-5.

[16] Chandra A, Bandyopadhyay AK, Bhaduri G. A comparative study of two methods of optic disc evaluation in patients of glaucoma. Oman Journal of Ophthalmol 2013;6(2):103-7.

[17] Danesh-Meyer HV, Ku JY, Papchenko TL, et al. Regional correlation of structure and function in glaucoma, using the disc damage likelihood scale, heidelberg retina tomograph, and visual fields. Ophthalmology 2006;113(4):603-11.

[18] Hornova J, Kuntz NJ, Prasad A, et al. Correlation of disc damage likelihood scale, visual field, and heidelberg retina tomograph II in patients with glaucoma. Eur J Ophthalmol 2007;18(5):739-47.

[19] Abdul Majid AS, Kwag JH, Jung SH, et al. Correlation between disc damage likelihood scale and optical coherence tomography in the diagnosis of glaucoma. Ophthalmologica 2010;224(5):274-82.

[20] Trastman-Caruso E, Jindal AP, Jadico SK, et al. Performance of the disc damage likelihood scale, OCT III, and HRT III in the diagnosis of open angle glaucoma. Investigative Ophthalmology \& Visual Science 2009;50(13):5816.

[21] Greaney MJ, Hoffman DC, Garway-Heath DF, et al. Comparison of optic nerve imaging methods to distinguish normal eyes from those with glaucoma. Invest Ophthalmol \& Vis Sci 2002;43(1):140-5.

[22] Spaeth GL, Henderer J, Steinmann W. The disc damage likelihood scale: its use in the diagnosis and management of glaucoma. Highlights Ophthalmol 2003;31(4):4-16. 